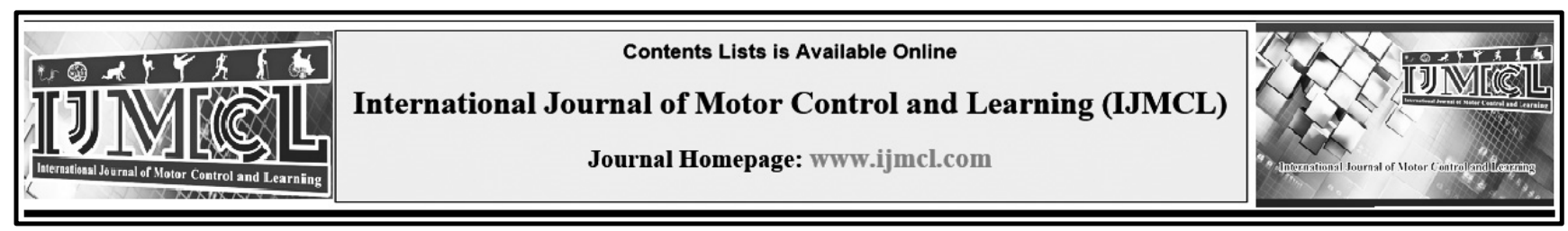

\title{
The Effect of Explicit and Implicit Learning on Static Balance
}

\author{
Gholam Hossein Nazemzadegan ${ }^{a^{*}}$, Mohaddeseh Yousefi ${ }^{\mathrm{b}}$

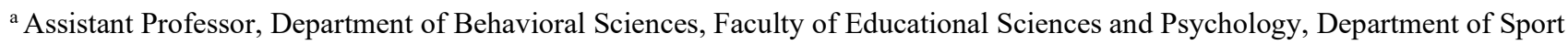 \\ Sciences, Shiraz University, Shiraz, Iran \\ ${ }^{\mathrm{b}}$ Master's Degree in Motor Behavior, Learning and Motor Control, Shiraz University, Shiraz, Iran
}

\begin{tabular}{l}
\hline Keywords \\
\hline Explicit Learning \\
Implicit Learning \\
Static Balance
\end{tabular}

Gholam Hossein Nazemzadegan, Email: ghnazem@gmail.com

Received: 2020/07/18

Accepted: 2020/10/19

Published: 2020/11/05

\begin{abstract}
Objective: The purpose of this study was to investigate the effect of learning type on balance.

Methods: For this purpose, 80 female students aged 9-12 years who were selected by random cluster sampling and after Flamingo test, they were divided into two groups randomly: explicit learning and implicit learning. The subjects were required to maintain balance on the Stabilometer. The acquisition stage included 24 attempts 60 seconds. Explicit learning groups received feedback while on the device, but the implicit learning group was required to perform a secondary cognitive task in addition to maintaining a balance without receiving feedback.

Results: Analysis of covariance with a significant level of $\mathrm{p} \leq 0.05$ showed that there was no significant difference between explicit and implicit learning in static balance.

Conclusion: The results indicate that the subjects of both groups had improvement in their post-test balance records compared to the pre-test. Therefore, probably the static balance with regard to its subcortical mechanism cannot be influenced by the type of learning.
\end{abstract}

\section{Introduction}

Balance is one of the main factors in performing most daily activities as well as an important indicator in determining the ability of athletes. This factor is defined as the ability to maintain or reverse the center of gravity in the range of reliance on, or control and maintain the condition of the body in space, in order to achieve sustainability and orientation (Shumway et al., 2001). Maintaining balance is one of the most important abilities that can be effective in human movement from childhood to old age. Changes in balance are affected by the conditions that the body moves from one to another position in relation to the environment. The information used to control the balance can be influenced by various factors, including the type of learning

Research has shown the effect of learning in sport skills. Shia et al. (2001) found that the effect of implicit learning in balance task acquisition, is different from the explicit. Explicit learning is a type of learning that a person is consciously learning skill or function. In this type, the learner is fully aware of the act of learning and is deliberately 
trying to improve his / her level of learning more and better. While implicit learning is another type of learning that a person is unconsciousness to learning skill and is in the face of explicit learning. In fact, the individual is unaware of what he/ she is learning (Taylor \& Bond, 2015). Investigating the effect of implicit and explicit learning on motor skills such as balance assignments and control posture is subject to different studies and are interesting to many researchers. Among these studies, a study by AJ Orl et al. (2006) pointed out that explicit and implicit learning in a balance task. This study included 42 students with an average age of 29 years who were divided into explicit and implicit learning groups. According to the type of learning, exercises with cognitive instructions and assignments were given to the groups and after two weeks, the transfer test was taken. The result obtained from this study was that the balance functions that were associated with verbal assignments were better than the equilibrium performance alone.

Jonathan et al. (2017) conducted a research on the walking of the elderly on treadmill and divided into two groups of internal concentration training (implicit learning) and external focus (explicit learning). Findings showed that implicit learning is more suitable for improving motor stability in the elderly. Maxwell et al. (2017) compared the ability of motor and learning skills in children using explicit and implicit approaches. The statistical population of the study was 261 children aged 9 to 12 who were divided into two groups of high and low motor ability according to the motor skills score in the upper and lower levels of golf skill. The results show that for children with low motor ability, implicit learning was beneficial, while it was better for children with high learning abilities to learn more explicitly. Abdoli et al. (2004) conducted a study titled "Comparison of explicit and implicit learning effects on chain reaction time". The results showed that both explicit and implicit participants participated in implementing a chain reaction time assignment, but no significant difference was found in the type of learning. Nejati et al. (2012) conducted a comparative study of explicit and implicit motor learning in dominant and non-dominant hands among young people. The results showed that the learning of the explicit and implicit movement of the dominant and dominant hand of young people is the same.

In fact, the antonym results of previous studies on the kind of learning on balance and determine the effect of learning on functional abilities such static balance are reasons for doing this study.

\section{Method}

After obtaining the necessary permissions, sampling from the students began and according to the Waterloo questionnaire, the superiority of the legs was determined, and parents' consent form was given to cooperate with them. First measurements including height and weight and the demographic questionnaire of each subject was completed by their parents. After recording personal data, the stages of the process began as follows:

The first stage was the pre-test, which included static balance test (flamingo). To perform the testT 
subjects should stand on a $50 \mathrm{~cm}$ long wooden, 3 $\mathrm{cm}$ wide, $5 \mathrm{~cm}$ high, with $15 \mathrm{~cm}$ retaining supports, covered with $4 \mathrm{~mm}$ foam baroque, and, as far as possible they could balance (Three times with a superior leg), the average of every 3 times from a test was recorded as the final record. The reliability of this test is higher than the ground and by Cronbach's alpha test for girls was 0.72

According to the results and records obtained in the pre-test phase, the number of subjects, which was 80 , was homogeneous in two groups of explicit and implicit learning. A balance device was used to carry out training efforts. Before the start of the attempts, the method of placement and how to control the balance was explained to the subjects, and then once on the balance machine, and the supplementary explanations were told to them, and after the subject's preparation, time began to record, which took 60 seconds to maintain balance on the balance machine. The device was purchased from the Satire Engineering Company, which has been used in many studies. The efforts of the implicit learning group were as follows. When the subject was placed on the machine, as long as he was mindful of the balance of the device, he had to review the six digits that were told at certain intervals and remember them. After the time of the machine, the figures were given to the researcher stated that, therefore, in this group, subjects were subconsciously maintaining balance by virtue of their mental functions and focusing on the figures, which led to learning how to better balance (implicit learning). Because in this group there is no other explanation in how to balance when placing on the balance machine, the subjects were not given to individuals and each subject must obtain balance control skills. The attempts of the explicit learning group were also such that the subject, before placing on the balance instrument and, when placed on it, needed to improve the balance of balance and, given the device's LEDs, they also received quick feedback so that if the balance device was oscillating, it was red and an indication of the error in maintaining the balance, and if it returned to a balanced state, it was a green light. The researcher also reminded them, if needed, that they would correct the balance. At this stage, each person should be placed on the device 24 times, and he would train his group's efforts. After each run, they rested for 2 minutes to prepare for the next attempt. Eventually, the average records of every 24 times were considered as the final record of the exercise effort.

In the third week, which was the post-test, all subjects retested the pre-test tests and the results were recorded to review the changes.

\section{Results}

In order to examine whether the type of learning can affect balance, one-way covariance analysis was used by considering pre-test as a covary factor which showed that the type of learning is not affect on the balance, ( $F=37 / 0$ and $p \leq 0.05)$; Therefore, according to the results, there is no significant difference in the static balance with the explicit and implicit learning exercises. 
Descriptive findings related to demographic variables of participants, mean and standard deviation of age, weight and height of subjects in groups learning are presented in Table 1.

Table 1. Mean and standard deviation of the indexes of the research groups.

\begin{tabular}{|c|c|c|c|}
\hline $\begin{array}{l}\text { Variable } \\
\text { Group }\end{array}$ & $\begin{array}{l}\text { Age } \\
\text { (years) }\end{array}$ & $\begin{array}{c}\text { Weight } \\
\text { (kilogram) }\end{array}$ & $\begin{array}{c}\text { Height } \\
\text { (centimeter) }\end{array}$ \\
\hline Explicit learning & $\begin{array}{c}10.62 \\
.64\end{array}$ & $\begin{array}{l}43.73 \\
11.73\end{array}$ & $\begin{array}{c}146.47 \\
10.14\end{array}$ \\
\hline Implicit learning & $\begin{array}{c}10.48 \\
.59\end{array}$ & $\begin{array}{c}38.66 \\
8.74\end{array}$ & $\begin{array}{c}144.32 \\
9.71\end{array}$ \\
\hline
\end{tabular}

In Fig. 1, the average static balance between the groups is presented in the pre and post-test phases. as we can see, the average in both groups in the post-test phase is higher than the pre-test stage, which indicates the effect of the training efforts in the subjects in the groups.

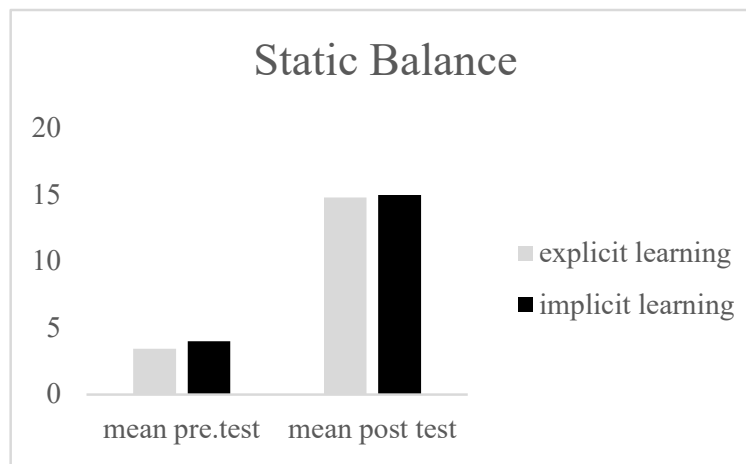

Figure 1. Average static balance of groups.

\section{Discussion and Conclusion}

The overall aim of this study was to compare the effect of explicit and implicit learning on static balance. The results in both groups showed an improvement in their post-test balances in comparison with the pre-test. But, in the end, there was no significant difference in the explicit and implicit learning in performing static balance. While, due to the similarity of the mean of groups in the post-test, the standard deviation of the hidden learning group was lower, which indicates a better effect of this type of learning.

The findings of this study were consistent with Nejati et al. (2012) and Abdoli et al. (2004) results. It showed that balance efforts lead to improvement of balance, but the effect of learning type on balance were the same. In the other hand our finding is divergent with J. Orle (2006), Jonathan (2017) and Maxwell (2017) results. The causes of inconsistency include the measurement tool and the training protocol.

Finally, according to the findings of this study, static balance cannot be influenced by the type of learning due to its subcortical mechanism. Thus, the effect of explicit and implicit learning on the static balance are the same. It is suggested that, given the importance of explicit and implicit learning, one should compare the types of learning to other abilities such as eye hand coordination or dynamic balance that are not use subcortical control to doing the task. As well as researcher can using the same method with different techniques to measure the balance. The findings of this study can be used to improve the level of performance and balance of students and adolescents. 


\section{References}

1. Abdoli, B., Ashayeri, H., Bagherzadeh, F., \& Farrokhi, A. (2004). Comparison of the effect of hidden and explicit learning on chain reaction time. Motion Magazine, 19.

2. Bond, K. M., \& Taylor, J. A. (2015). Flexible explicit but rigid implicit learning in a visuomotor adaptation task. Journal of neurophysiology, 113(10), 3836-3849.

3. Maxwell, J. P., Capio, C. M., \& Masters, R. S. (2017). Interaction between motor ability and skill learning in children: application of implicit and explicit approaches. European journal of sport science, 17(4), 407-416.

4. Nejati, Vahid, Izadi Najafabadi, Entezari. (2014) Comparative study of explicit and implicit motor learning of dominant and non-dominant hand in young people. Jundishapur Medical Journal (12) 2, 187-177.

5. Orrell, A. J., Eves, F. F., \& Masters, R. S. (2006). Implicit motor learning of a balancing task. Gait \& posture, 23(1), 9-16.

6. Shea, CH., Wulf, G., Whitacre, CA. and Park, JH. (2001). Surfing the implicit wave. The Quarterly Journal of Experimental Psychology: Section $A$. 54(3):841-62.

7. Shumway-Cook, A., \& Woollacott, M. H. (2001). Theory and Practical Applications. 